\title{
A Comparative Study of Free-Viewpoint Video Techniques For Sports Events
}

\author{
JJM Kilner, J Starck, A Hilton \\ Centre For Vision, Speech and Signal Processing, University of Surrey, United Kingdom, \\ $\{$ J.Kilner, J.Starck, A.Hilton $\} @$ surrey.ac.uk
}

Keywords: free-viewpoint video, scene reconstruction, quantitative analysis, quality, sports

\begin{abstract}
This paper presents a quantitative analysis of free-viewpoint video techniques applied to the problem of virtual view synthesis in sports events. A consideration of errors in the synthesis pipeline is presented along with a taxonomy of these errors and a framework for evaluating the quality of view synthesis when compared to ground truth. Three reconstruction techniques are evaluated, billboarding, shape from silhouette and view-dependent shape from silhouette. View-dependent rendering is used for virtual view synthesis. It is shown that currently the shape from silhouette technique provides the best completeness, while the view-dependent shape from silhouette technique provides the best appearance.
\end{abstract}

\section{Introduction}

In traditional fixed-viewpoint video an event is recorded from a specific location using a camera. When the video is rendered the viewpoint it presents is that of the camera that was used. Some mobility of viewpoint can be achieved by combining multiple fixed-viewpoint video streams and switching between them, but the viewpoint is still fundamentally limited to locations that are co-incident with a physical camera that was used to record the event. Free-viewpoint video (FVV) attempts to break this restriction by allowing the specification of the camera location at the point of rendering rather than at the point of recording.

\subsection{Free-Viewpoint Video for Sports}

Television broadcasts of sporting events command large audiences. As a result broadcasters seek to give viewers the most entertaining coverage and the most insightful commentary to gain the largest market share. As showing what happened during a sporting event in as much detail as possible is key to both of these aims, it can be seen that there are several applications for FVV in the sporting arena.

Sports often rely on the movement of contestants within a specific playing area, with different rules applying to different regions of that area. Often cameras are set up to try to get a direct view of the boundaries so that the viewer can see clearly whether a rule of the game was infringed. However, as the broadcaster typically has a limited number of cameras, it is necessary to place the majority of them so that interesting coverage of the whole of the event can be provided, not just action around the boundaries. As a result the broadcaster often has to attempt to preempt where the interesting boundary events will take place. If they get this wrong they risk missing out on crucial footage of the event, and also waste a camera which could have been providing another angle for general coverage.

A great deal of tactical interest in sport relies on the spacial configuration of the competitors. Tactics can be inferred from relationships as simple as the relative positions of competitors in a race, to those that are considerably more complex, such as the relationship that determines whether a player is offside in football. These relationships are often very difficult to determine unless a camera is specifically placed so that it highlights the desired relationship. As the configuration of the contestants may become interesting at an arbitrary time and location in the playing area, it is impossible to place cameras accurately to guarantee a good viewpoint. As a result it can often be very difficult, if not impossible, to work out the spacial relationship of the contestants from the broadcast coverage of a sporting event.

Finally, with sports broadcasters competing for share of the large audiences that big sporting events command, there is a demand for special effects such as the "matrix style" camera transitions seen at the Super Bowl [7]. Given these considerations, and the fact that the location of cameras may be further constrained by the shape of the arena the event is taking place in, crowd seating, etc, it can be seen that an FVV system suitable for use in sporting events is highly desirable as it allows broadcasters to generate the output they want without having to preempt the correct camera locations for every possible eventuality.

\subsection{A Comparative Study}

Most of the current techniques for FVV are designed around a multi-camera studio environment with controlled lighting and well-calibrated static cameras. It is unsurprising therefore to find that they do not perform at an acceptable quality in the context of outdoor sports coverage. However, the techniques differ in the extent to which they are affected by the different 
issues. Thus it is not a simple case of choosing the "best" technique, as a direct comparison tells us little. Instead a comparison must be undertaken which classifies the error in the techniques, which errors are fundamental and which can be avoided, and the extent to which different errors affect the quality of the final result. Thus a comparative study is required to show the effect of employing the different techniques on the quality of the synthesised video.

In this paper we will perform a quantitative analysis of several techniques as applied to an outdoor broadcast of a sports event. Section 2 discusses the background to the problem, covering issues of reconstruction and rendering as applied to FVV, and an analysis of the proposed applications of FVV in the sports domain. Section 3 then presents an analysis and taxonomy of the errors that can occur in the generation of a synthetic video sequence. Section 4 presents the quantitative analysis employed, Section 5 the results of this analysis, and Section 6 a discussion of these results. Section 7 presents the conclusions drawn from the analysis.

\section{Background}

Free-viewpoint video has received considerable interest within the studio environment. The Virtualized Reality system [10] first used 51 cameras distributed over a $5 \mathrm{~m}$ dome to capture the performance of an actor in a studio. Multiple-view silhouettes [18, 15, 3] and photo-consistency across views [22] have been used for view synthesis with a reduced number of cameras. Video-rate reconstruction using silhouettes has been achieved using an image-based representation [15] and probabilistic approaches [8] have been proposed to improve reconstruction in the presence of inexact foreground segmentation. High-quality view synthesis has now been demonstrated by integrating stereo reconstruction and matting for short-baseline camera positions [23] and by deriving a view-dependent geometric representation with wide-baseline cameras [20].

Only limited work to date has addressed the problem of free-viewpoint video synthesis in external environments for sports events. Image morphing has been applied for view interpolation with weak camera calibration [5, 9] and a simplified geometric representation using planar billboards has been proposed for real-time view synthesis [11]. High-quality view synthesis from wide-baseline camera positions in an uncontrolled external environment remains an open and challenging problem.

\subsection{Reconstruction Techniques}

Different techniques for scene reconstruction have different overheads and trade-offs in terms of quality and fidelity. For the proposed application of sporting event reconstruction, realtime playback capabilities along with the ability to work on data from sparse view-points are important properties. This has led to three possible alternatives: billboards [11], visual hull [21], and the view-dependent visual hull [16].

In billboarding a single polygon is placed co-incident with the object that it represents. This polygon is then rotated around an axis or point (typically the $\mathrm{Y}$ axis) so that it retains its original position, but is constantly facing the virtual camera. An image of the original object is then applied to the polygon as a texture map. This technique can often give good results with very little overhead as large-scale parallax effects are handled by the relative positioning of the billboards, while the lack of small-scale parallax is often not noticed. For the billboarding technique discussed in this paper we have located the objects in each image using a simple segmentation method, and have then triangulated each object's position. A billboard is then placed at these triangulated locations and assigned a set of texture maps by projecting the billboard to each camera in turn and extracting the relevant section of the image. In this way a simple geometric representation of the scene along with relevant texture maps can be built up.

The visual hull [13] derives scene geometry that is consistent with a set of image silhouettes. Reconstruction projects the silhouettes of the foreground objects in each image using the calibration data for the relevant camera. It then finds the intersections of these projections. Each intersection defines the largest possible surface that could produce the silhouettes in the original images. A volumetric approach is adopted [21] that divides space into a voxel grid and back-projects every voxel to each image. By comparing the overlap of the voxel to the silhouettes for each camera you can determine the voxel's occupancy. Overlap can be tested up to a given reprojection error to account for calibration errors in the camera system. The surfaces generated by the voxels are then triangulated using the marching cubes algorithm [14] to produce a mesh.

Billboarding and the visual hull are compared to view synthesis using the view-dependent visual hull (VDVH) [16]. Reconstruction derives a view-dependent 2.5D depth representation for the visual hull with respect to a given viewpoint. Surface geometry is derived in the image domain by reprojecting a ray from this given viewpoint and deriving the exact intersection with each image silhouette to provide a depth-per-pixel. The VDVH is used to provide a multiple $2.5 \mathrm{D}$ image plus depth representation of the scene with respect to each camera view for virtual view synthesis.

\subsection{Free-Viewpoint Video Rendering}

In the field of rendering for FVV two classes of data are used. Global data is invariant with the virtual camera position and orientation. In contrast view-dependent data relies on 
the virtual camera location, either to choose from one of several data sets, or directly for its computation. When rendering the reconstruction, the above distinction gives us three different classes of rendering technique: global geometry and texture, global geometry and view-dependent texture, and view-dependent geometry and texture. FVV techniques make use of view-dependent rendering to overcome inaccuracies in scene reconstruction from a limited number of camera views. View synthesis is performed either by sampling appearance from a subset of cameras closest to a virtual view and hence minimising the error from inexact correspondence [6], or by deriving a view-dependent geometry for rendering [20] to optimise the correspondence in the camera set used in rendering.

The term view-dependent texturing was introduced by Debevec et al. [6] and refers to the technique of choosing between multiple texture maps to apply to a surface based on the orientation of the virtual camera relative to the surfaces of the mesh. Often multiple close textures are chosen and are blended on the surface with their blend weights dependent on the angular distance between the viewing rays of the virtual camera and the viewing rays of the original cameras that were used to generate the original images being used as texture maps. Applying a view-dependent texture to a static mesh provides a technique that uses global geometry and view-dependent texture. The visual hull technique that we are evaluating falls into this class of rendering.

Finally you can modify both the geometry and the texture based on the viewing angle. This extends the concept of view-dependent texturing into the geometry domain and entails choosing the geometry to display (along with the texture to apply) based on the virtual camera location. This is the rendering strategy adopted by the VDVH technique [17] included in this comparison which selects the most relevant meshes and draws them in back-to-front order. Another member of this class of technique is where the geometry is computed based on the location of the virtual camera. In its simplest form this can give us what is known as billboarding. This is where a single polygon is rotated so as to keep facing the virtual camera, and onto that billboard a texturemap of the object is projected. The billboarding technique considered in this paper uses view-dependent geometry and texture as described above.

\subsection{Applications in the Sports Domain}

As mentioned before there are two main applications of FVV in the sports domain. The first is that of post-event analysis, the second is camera virtualisation (special effects such as transitions are just an extreme example of this kind of application)[7].

For post-event analysis, the overriding concern is
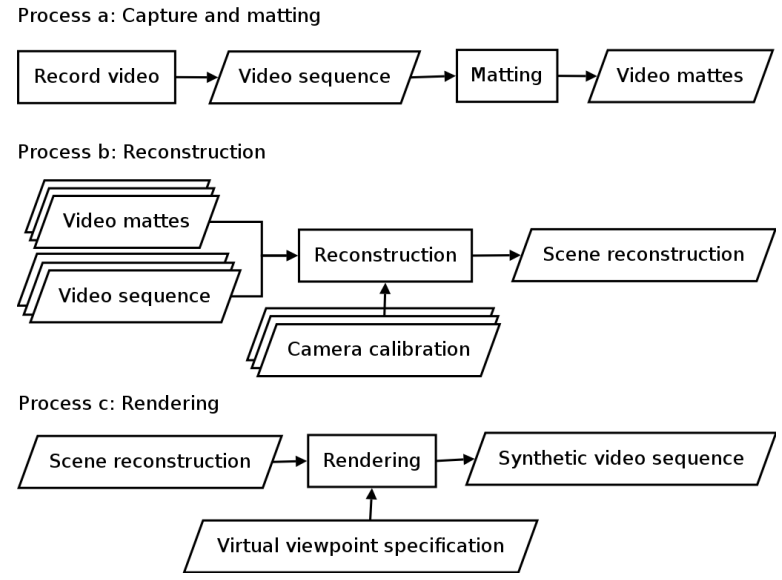

Figure 1: The FVV synthesis pipeline.

reconstruction accuracy. For a tactical analysis of a sporting event to be performed it is crucial that the tactics of the situation can be accurately determined. If a player is rendered in accurate detail, but in an incorrect location then incorrect conclusions can be drawn from the synthetic video. This means that while a visually pleasing representation is desireable in this application, it must not be obtained at the expense of accuracy. An example of this can be seen in the Pierot system [1]. In this system, a player's location is estimated by intersection with the ground plane of the pitch and the player is represented simply as a billboard projected onto an artificial backdrop. The lack of visual quality inherent in this simple synthetic view is not important as the quality is sufficient to represent the situation and the player locations.

The application of camera virtualisation however has very different constraints $[9,11]$. With this type of reconstruction the aim is to generate a synthetic video stream from the data captured by the existing cameras. The resultant reconstruction does not have to be as accurate, however the visual quality of the synthetic video produced must be high. For example, a typical application might be to allow a virtual tracking shot between two real cameras, in this case the absolute positions of objects does not need to be correct, as long as their relative positions are correct enough to give the required parallax. These two applications thus exert similar but distinct selection criteria for the reconstruction/rendering technique to choose.

\section{A Taxonomy of Errors}

The FVV synthesis pipeline consists of the set of steps shown in Figure 1. In the capture and matting process, a video sequence is captured using a video camera and processed so as to extract mattes of the images in the sequence. A matte is a single per-pixel value, stored as either a monochrome bitmap or an extra channel in the image. The per-pixel value indicates how much of that pixel is foreground and how much 
(a)

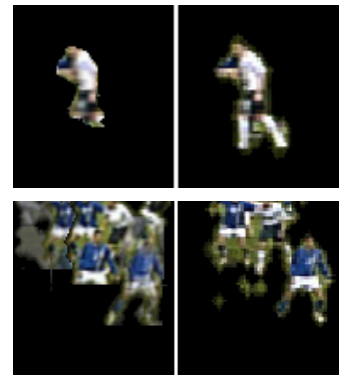

(b)

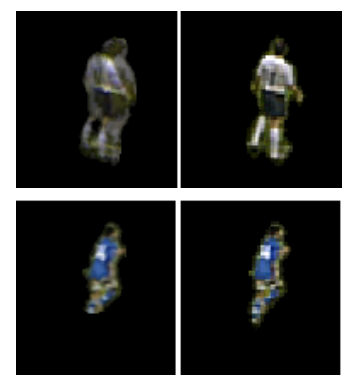

Figure 2: A comparison of synthetic images to their corresponding ground truths. (a) shows an incomplete synthetic image, (b) shows a synthetic image where the player is incorrectly rendered twice. (c) shows a player incorrectly rendered to a foreground region and (d) shows a blurred player.

is background, with intermediate values indicating a pixel that is partially foreground and partially background. During reconstruction the images, mattes and camera calibrations from all cameras are combined and a scene reconstruction is generated. During rendering the reconstruction is combined with the specified virtual viewpoint and a synthetic video sequence is generated. In the implementation presented in this paper, the capture and matting process is shared between all reconstruction techniques and an implementation of the rendering process has been developed that can render the reconstructions generated by any of the reconstruction techniques.

The errors inherent in this process can be classified by considering the ways in which a synthetic video sequence generated for a given viewpoint can differ from the video sequence that would have been captured by a real camera placed at that viewpoint. In the following discussion the frame from the synthetic video sequence will be referred to as $I^{\prime}$ and the corresponding image from the real camera will be referred to as $I$. Similarly $R^{\prime}$ is a region in $I^{\prime}$ which corresponds to the region $R$ in $I$.

\subsection{Errors in Shape and Appearance}

Errors in shape are errors where $I^{\prime}$ is missing a foreground element that is present in $I$, or $I^{\prime}$ contains an extraneous foreground element that was not present in $I$. Examples are missing limbs or double images as shown in Figure 2 (a) and (b). In both cases pixels in $I^{\prime}$ have been incorrectly classified as either foreground or background. This error can also be considered an error of completeness where the reproduction of the foreground is incomplete or overcomplete. Foreground regions in $I^{\prime}$ which are free from errors of shape are regions of correct shape.

Errors in appearance occur when a region of correct shape $R^{\prime}$ contains different pixel values to $R$. This error can occur in two ways. If a surface whose reconstruction is rendered to $R^{\prime}$ is not the surface whose image appears in $R$ then an error of appearance occurs. Alternatively, if the correct surface is rendered but the reconstruction technique has incorrectly synthesised the view of the surface, $R^{\prime}$ will be a distorted version of $R$. Examples are the rendering of surfaces in incorrect locations and blurred rendering of surfaces, as shown in Figure 2 (c) and (d). In both cases pixel values in the generated image are inconsistent with the correct pixel values. A region of correct shape that is free from errors in appearance is a region of correct view synthesis.

Table 1 summarises these classifications. This taxonomy covers all possible errors in the domain of synthesised foreground images. Through the distinction between foreground and background (and hence between shape and appearance), a measurement of image fidelity can be composed that conveys more information about the errors in the synthesis pipeline. If the entirety of $I^{\prime}$ is treated as a region of correct shape then this analysis reduces to a comparison of pixel values across the image. This relates the classification to standard image quality measurements which are measures of appearance across the entire image. It is the distinction between foreground and background which allows us to also consider shape in our analysis.

\subsection{Temporal Errors}

The synthetic images generated for FVV are not being created in isolation, but form part of a temporal sequence that is the synthesised video stream. The stability of errors in the sequence can have a significant influence on the perceived quality of the video. For example three errors occurring in consecutive frames may give a better visual appearance than all three errors occurring in the same frame, although the total error in the sequence is the same. Therefore it is as important to watch the rate of change of error across a sequence as it is to look at the absolute error values for any given frame in order to determine the quality of the synthetic video.

\section{Quantitative evaluation}

Three measures were used to gauge the visual quality of the synthetic video. These measures were computed across the whole image (full reference techniques). The measures used were similarity in shape, similarity in appearance and the peak signal-noise ratio. In the following discussion, $I$ refers to the original image and $I^{\prime}$ refers to the synthesised image. Both images contain $n$ pixels. $p$ and $q$ are pixels in $I$ while $p^{\prime}$ and $q^{\prime}$ refer to pixels in $I^{\prime}$.

An $r$-shuffle is a perturbation of an image such that if $I^{\prime}$ is an $r$-shuffle of $I$ then every pixel $p$ will be transformed to a pixel 


\begin{tabular}{|c|c|c|c|}
\hline Error & Image in $R^{\prime}$ & Image in $R$ & Classification \\
\hline $\begin{array}{l}\text { missing foreground } \\
\text { extraneous foreground } \\
\text { none }\end{array}$ & $\begin{array}{c}\text { present } \\
\text { absent } \\
\text { present }\end{array}$ & $\begin{array}{l}\text { absent } \\
\text { present } \\
\text { present }\end{array}$ & $\begin{array}{l}\text { error in shape } \\
\text { error in shape } \\
\text { correct shape }\end{array}$ \\
\hline $\begin{array}{l}\text { incorrect image } \\
\text { distorted image } \\
\text { none }\end{array}$ & $\begin{array}{c}\text { image of } \beta \\
\text { distorted image of } \alpha \\
\text { image of } \alpha\end{array}$ & $\begin{array}{l}\text { image of } \alpha \\
\text { image of } \alpha \\
\text { image of } \alpha\end{array}$ & $\begin{array}{l}\text { error in appearance } \\
\text { error in appearance } \\
\text { correct view synthesis }\end{array}$ \\
\hline
\end{tabular}

Table 1: Classification of errors in foreground synthesis.

$p^{\prime}$ such that $\left|p^{\prime}-p\right|<r$ [12]. Let $I_{p}^{\prime}$ be the image that would be generated by a perfect rendering of a perfect reconstruction of a scene. Due to the accumulation of errors in the reconstruction process the generated image $I^{\prime}$ will be a distortion of $I_{p}^{\prime}$. By modelling this distortion as an $r$-shuffle, the accumulation of errors in the view synthesis pipeline can be compensated for, allowing an assessment of the true fidelity of the underlying reconstruction technique.

The function $b(p)$ determines whether the pixel $p$ in an image is part of $B$ the set of background pixels in the image. In our implementation $b(p)$ is defined as the Kronecker delta function applied to the intensity of the pixel $\nu(p)$.

$$
b(p)= \begin{cases}1 & (\nu(p)=0) \\ 0 & (\nu(p) \neq 0)\end{cases}
$$

The set of pixels containing foreground elements $F$ is then defined such that $F \cap B=0$ and $F \cup B=I$. The function $f(p)$ which determines whether pixel $p$ is a member of $F$ is then simply defined as the inversion of $b(p)$

$$
f(p)=1-b(p)
$$

The $r$-neighbourhood $N_{r}$ of a pixel $p$ on the image $I$ is defined such that for some other pixel $q$

$$
q \in N_{r}(p) \text { if and only if } q \in I \text { and }|q-p|<r
$$

Combining equations 2 and 3 we can define the function $s$ which determines the best match of shape between the pixel $p$ and some pixel in the region $N_{r}\left(p^{\prime}\right)$.

$$
s\left(p, p^{\prime}\right)=f(p) * \arg \max _{q}^{\prime}\left(f\left(q^{\prime}\right)\right), q^{\prime} \in N_{r}\left(p^{\prime}\right)
$$

Performing a summation over the entire image and normalising by $F \cup F^{\prime}$ defines the function which calculates the maximum possible similarity in shape between the images $I$ and $I^{\prime}$.

$$
S\left(I, I^{\prime}\right)=\frac{\sum_{i=1}^{n} s\left(p_{i}, p_{i}^{\prime}\right)}{\sum_{i=1}^{n} \max \left(f\left(p_{i}\right), f\left(p_{i}^{\prime}\right)\right)}
$$

The appearance matching function $a\left(p, p^{\prime}\right)$ compares the colour value of two pixels. If the colour value of a pixel in RGB space is given by $\kappa(p)$ then $a\left(p, p^{\prime}\right)$ is defined as

$$
a\left(p, p^{\prime}\right)= \begin{cases}1 & \left(\left|\kappa(p)-\kappa\left(p^{\prime}\right)\right| \leq \tau\right) \\ 0 & \left(\left|\kappa(p)-\kappa\left(p^{\prime}\right)\right|>\tau\right)\end{cases}
$$

\begin{tabular}{|c|c|c|c|c|}
\hline Image & Shape & Appearance & PSNR & VIF \\
\hline Original & 1 & 1 & inf & 1 \\
Median & 0.99 & 0.98 & 73.52 & 0.36 \\
Blur & 0.75 & 0.96 & 72.81 & 0.32 \\
VH & 0.86 & 0.99 & 70.19 & 0.22 \\
VH - 1 & 0.84 & 0.95 & 68.08 & 0.15 \\
BB & 0.81 & 0.95 & 67.12 & 0.17 \\
BB - 1 & 0.70 & 0.86 & 65.11 & 0.08 \\
VDVH & 0.56 & 0.91 & 65.05 & 0.07 \\
VDVH -1 & 0.56 & 0.91 & 64.88 & 0.06 \\
Blank & 0 & 0 & 62.17 & 0 \\
\hline
\end{tabular}

Table 2: Comparison of evaluation techniques on a single frame.

where $\tau$ is some chosen small threshold. Integrating over the entire image and normalising by $F \cap F^{\prime}$ gives the function which measures the similarity of appearance between the two images

$$
A\left(I, I^{\prime}\right)=\frac{\sum_{i=1}^{n} \arg \max _{q_{i}^{\prime}} a\left(p_{i}, q_{i}^{\prime}\right) s\left(p_{i}, p_{i}^{\prime}\right)}{\sum_{i=1}^{n} f\left(p_{i}\right) f\left(p_{i}^{\prime}\right)}, q_{i}^{\prime} \in N_{r}\left(p_{i}^{\prime}\right)
$$

The peak signal-noise ratio (PSNR) is given by

$$
\operatorname{PSNR}\left(I, I^{\prime}\right)=20 \log _{10}\left(\frac{K n}{\sqrt{\sum_{i=1}^{n}\left|\kappa\left(p_{i}\right)-\kappa\left(p_{i}^{\prime}\right)\right|^{2}}}\right)
$$

where $K$ is the maximum value that can be given by $\kappa(x)$.

Table 2 shows a comparison of these measures against the Visual Information Fidelity measure (VIF) [19] of visual quality in an image which was chosen as a baseline fullreference quality metric. The comparison was carried out on several test images, some consisting of filtered versions of an original image and others on reconstructions of the scene. It should be noted that for this test the "original" used was a hand-matted image, thus the reconstruction scores are particularly low as they include errors from matting. It can be seen that measures are in broad agreement, justifying the use 


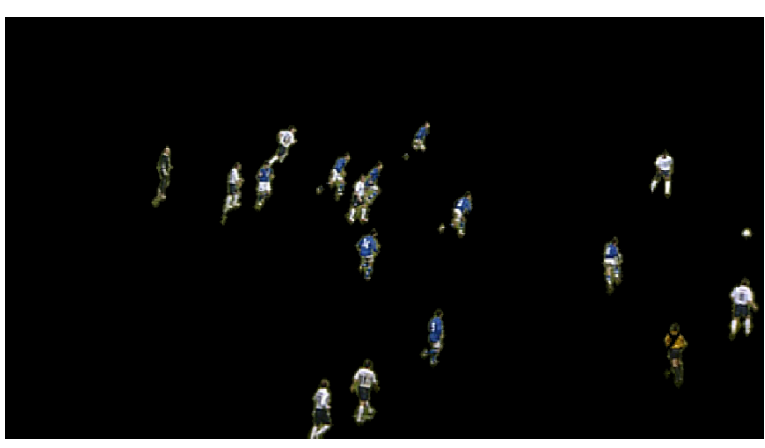

Figure 3: Ground truth image from camera 5.

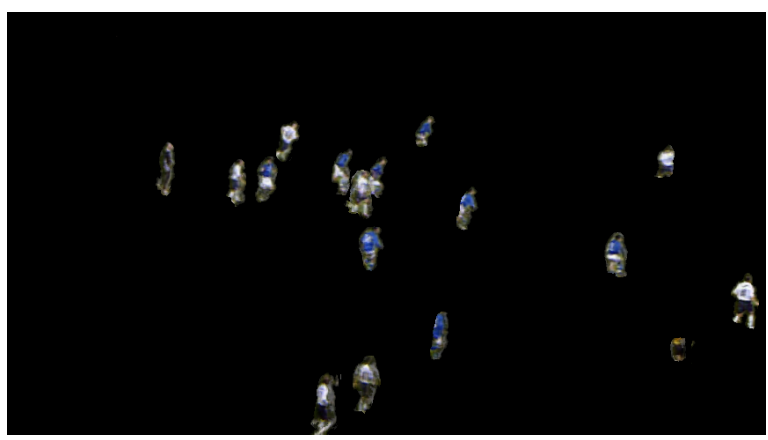

Figure 4: Visual Hull reconstruction not using camera 5 with compensation for a silhouette error of 3 pixels.

of these measures in the comparison, however the shape and appearance figures give more information than VIF or PSNR as to the nature of the reconstruction. For example all scores agree that the blur transformation produces a worse effect than the median filter, but the shape and appearance scores correctly indicate that the median filter preserves the shape of the image which the blur does not.

\section{Results}

The data set chosen for this evaluation was a recording of a football match. The recording was made with 15 static cameras arranged around 90 degrees of the stadium. This data set was chosen as stable calibration data is available for the cameras, and the arrangement of the cameras allows "leave one out" and "leave three out" comparisons without excessively reducing the quality of the reconstruction. This allows consideration of the behaviour of each technique as information becomes more limited.

The techniques of billboarding, visual hull, and VDVH were then compared. A single renderer capable of rendering all the scene reconstructions was created. This avoided differences in camera representation or lighting creating significant variation in global error between the techniques. A sequence of 100 frames of video from the 15-camera recording was matted using Bayesian matting [4]. Three experiments were then performed for each technique. In each experiment a reconstruction of the scene was generated using the original video sequence and mattes, and 100 frames of synthetic video produced using the camera calibration for camera 5. In the first experiment, all available data sets were used to reconstruct the scene (the "leave none out" test). In the second, camera 5 was omitted from the data set (the "leave one out" test) and in the third, cameras 4, 5 and 6 were omitted (the "leave three out" test). A ground truth video stream was then generated by combining the mattes and video for camera 5 and resampling this image to the correct aspect ratio while correcting for centre-point shift and radial distortion. The synthetic video streams were then compared with the ground truth video stream using the technique described in Section 4.

In the legends on the graphs accompanying this text "BB" refers to billboarding, "VH" refers to visual hull with viewdependent texture mapping, and "VDVH" refers to viewdependent visual hull. The suffix "-1" refers to the "leave one out" test and the suffix "- 3 " refers to the "leave three out" test.

The lefthand column of Figure 5 shows the behaviour of the shape scores for each of the techniques as the magnitude of the estimated system error is increased. The shape graph for the "leave none out" test shows that none of the techniques achieve a score of 1 , even when compensating for large system errors. Some of this is due to errors in the original matting as the visual hull techniques correctly discard areas of shadow that are incorrectly marked as foreground in the mattes. Most however are genuine view synthesis errors. All techniques discard small regions, which leads to small regions such as the football being incorrectly discarded from the reconstructions. Also, both visual hull and billboarding can clip players when camera calibration is inaccurate, leading to missing foreground sections.

It can also be seen that although the VDVH technique has a low score for all three experiments, it does not degrade as much as the billboard or the visual hull. This is because the VDVH generally reconstructs quite accurately, however it suffers quite extensively from missing and clipped players. These problems are not exacerbated by the removal of input cameras from the system, hence the degradation is not as great as might be expected. Finally it should be noted that although the billboard technique initially scores almost as well as the visual hull it is the technique which degrades most as cameras are removed from the system. This is expected as billboards, being the simplest geometrical representation of the scene, provide the worst interpolation as the distance between views increases.

The righthand column of Figure 5 shows the behaviour of the appearance scores for each of the techniques as the magnitude of the estimated system error is increased. The appearance graph for the "leave none out" test shows that where objects are rendered with no interpolation of view the appearance is preserved with high fidelity. When no error compensation is applied, the appearance score for all techniques is significantly 

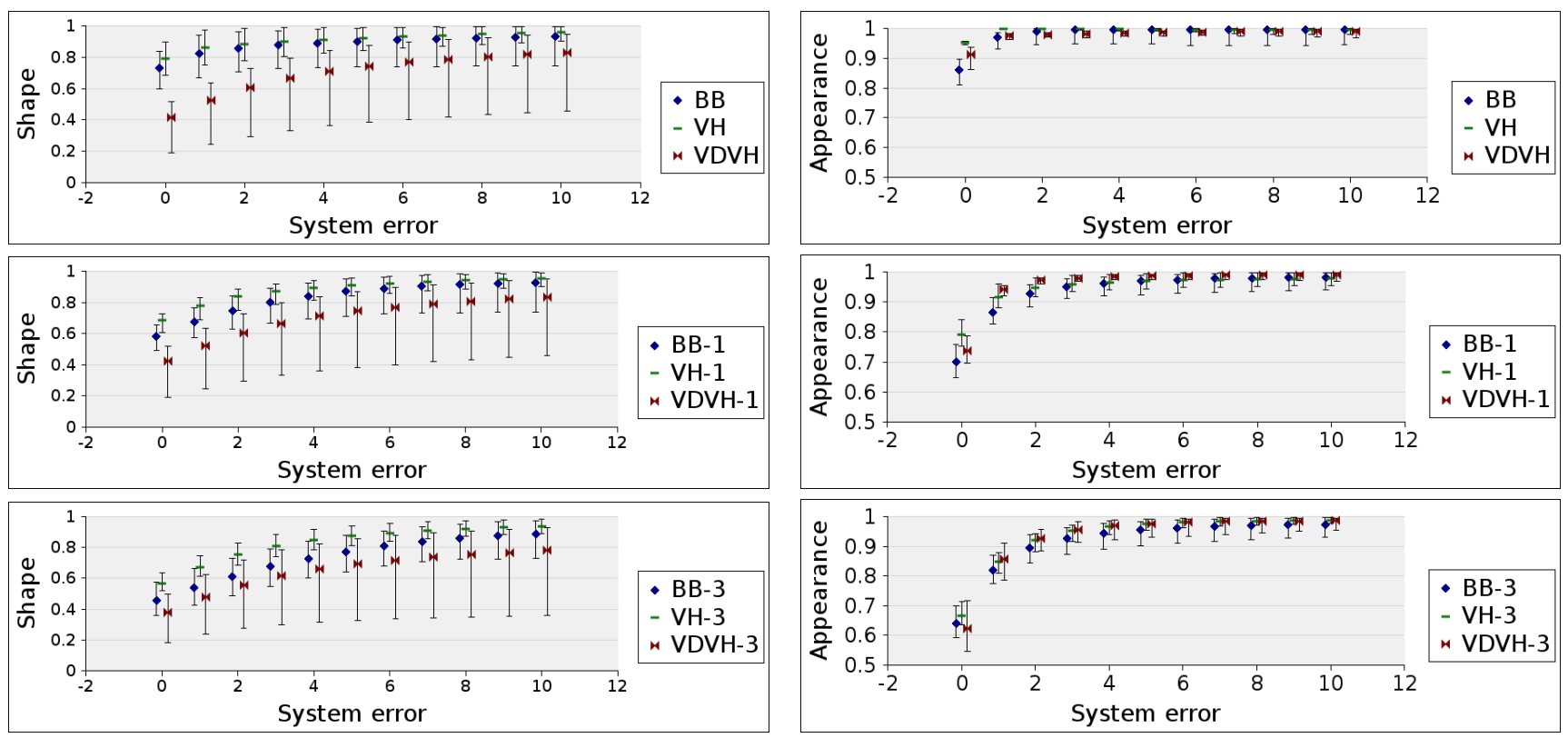

Figure 5: Graphs of shape and appearance scores vs. estimated system error. Shape is on the left, appearance on the right. The first row is the "leave none out" test, the second is the "leave one out" test and the third the "leave three out" test.

decreased. This is mostly due to resampling errors which occur when the original image is corrected for use as ground truth and when the camera images are converted to the appropriate format for use in the renderer. These errors are typically only of the order of a single pixel and hence the scores improve greatly once an error correction of 1 pixel is applied.

The data shows that when an error of a single pixel is allowed for in the "leave three out" experiment, the VDVH technique performs best, even though this same technique gives the worst shape scores. Finally it should be noted that even in this worst case "leave three out" test, appearance is generally synthesised with high fidelity compared to shape. The performance of the billboarding technique, which ignores small scale surface shape, indicates that the pressing problem with all current techniques is one of generating a scene reconstruction that is accurate and complete at the large scale; small-scale parallax having little effect on the appearance.

Figure 6 shows the rate of change of the shape score over time. When a defect appears or disappears it causes a reduction or increase in the shape score. By looking at the rate of change of the shape score, temporal artifacts such as players flickering in and out of visibility can be seen clearly. The graph shows that currently the VDVH technique suffers most from temporal artifacts in synthesised video.

Figure 7 shows the PSNR for each of the techniques in the three different experiments. The curves illustrate the degradation in overall image quality as the information provided for synthesis is reduced. It can be seen that the visual hull and billboarding techniques degrade in a similar manner, although

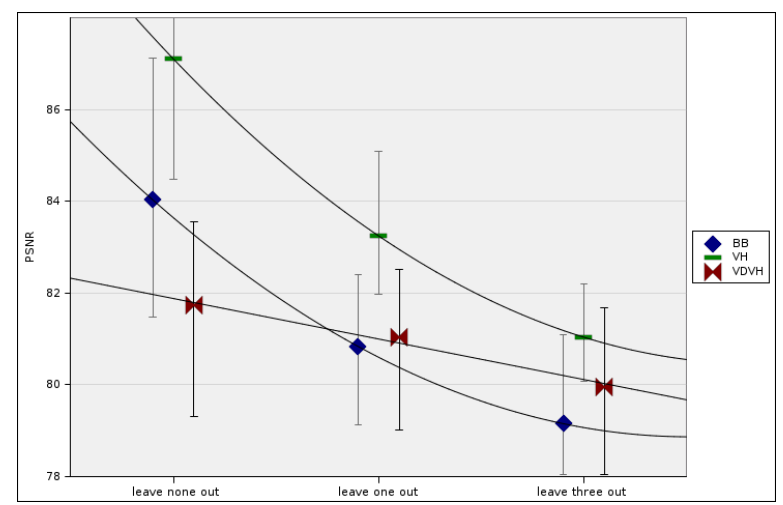

Figure 7: PSNR for the techniques across all tests.

billboarding is of a consistently lower quality. This is due to the effect of incoherence in the view-dependent texturing algorithm employed by both techniques. The view-dependent texturing algorithm applies a linear blend between the two images rendered to a polygon. The images chosen are the most relevant images from the source cameras. The blend weights applied to each image are related to the angular difference between the virtual view and the source camera for the texture. If the surface on which this blending occurs is not located accurately then the two images will not co-incide. As a result blurring and double-images will occur. This can be seen in both the visual hull and billboard synthesised images. As the disparity increases so does the effect of this blurring. As would be expected, the visual hull gives much better coherence between the images by mapping the textures on to a non-planar surface. This allows the combination of images with significantly different silhouettes as well as accounting 


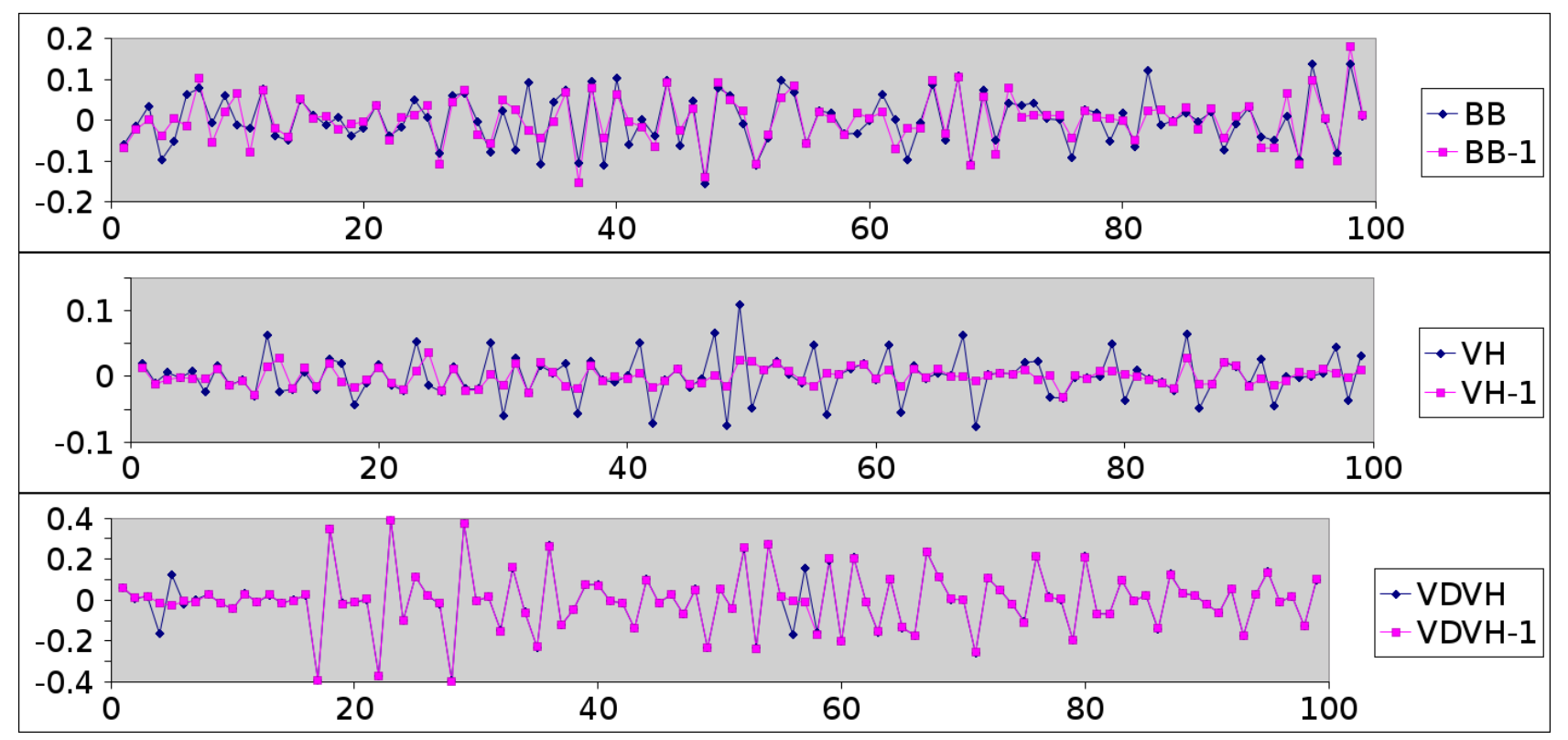

Figure 6: Rate of change of the shape score for several techniques over the 100 frames.

for small-scale parallax effects. This accounts for the better performance of visual hull. The VDVH technique does not use view-dependent texturing, instead a set of view-dependent meshes are generated and then rendered in back-to-front order. While this can still produce double images at the edges of the object, it avoids these artifacts in the region covered by the most relevant mesh, as the rendering technique overwrites the less relevant meshes. This accounts for the improved degradation in quality achieved by the VDVH as input cameras are reduced. As PSNR is a combined measure of shape and appearance over the entire image it can be seen that the low shape fidelity achieved by the VDVH accounts for the poor PSNR compared to the other two techniques.

\section{Discussion of Errors}

\subsection{Errors in shape}

Matting errors affect all of the techniques presented in this paper. The matting technique used is not perfect and errors at this stage will adversely affect all considered techniques. Matting error will particularly affect the visual hull techniques as they use a global representation which assumes coherent silhouettes between all cameras. The result of this is that the error due to matting in the visual hull of an object is the union of all errors in the matting of that object across all cameras. Thus small but different errors across the silhouettes can lead to large reconstruction errors. This error can be seen in Figure 2(a) where a player has been eroded due to matting errors in other views. Billboarding is the least susceptible to this error as the geometric representation is constant and so only the mattes being rendered as a texture map affects the rendered billboard's shape. The visual hull technique attempts to avoid this error by applying an error compensation strategy which reduces the size of regions that are cut away at the cost of generating inflated visual hulls.

Calibration error in the cameras will also affect all techniques. If the camera's calibration data is inaccurate, then the reconstruction will be inaccurate. Misalignment will lead to reduced visual hulls in the same way as they are produced by matting errors. If the reconstruction is inaccurate then items will be reconstructed incorrectly or will be reconstructed to incorrect locations. This will result in truncated objects or rendering to an incorrect location. This can be seen clearly in the billboarding technique where poorly calibrated cameras place the billboard in an incorrect location leading to truncation of the image projected onto the billboard as shown in Figure 8 (a).

The view-dependent texturing employed in rendering both the visual hull and the billboard reconstructions will generate double images if the textures that are being blended do not coincide well. This will occur when the surface being rendered is reconstructed in the wrong location. This can be seen in Figure 8 (b). While a correct visual hull will not display this problem, it is unavoidable in the case of billboards with wide baseline data sets, as often images with very different silhouettes may be presented which are impossible to blend with a linear blend as shown in Figure 8 (c).

The billboarding technique does not have any way to handle inter-object occlusions. When objects occlude each other in the source image, simple matting cannot separate these two objects, however a view from a different camera will separate the two objects leading to two billboards being created. Often 

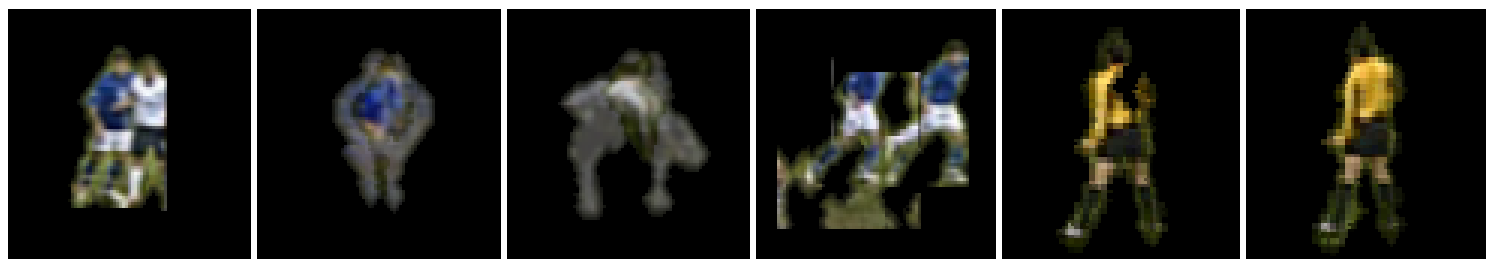

Figure 8: Examples of errors in image synthesis. Images a-f run left to right.

part of the furthest object will appear in the near billboard as well as in the far billboard, leading to the object being rendered twice as shown in Figure 8 (d).

\subsection{Errors in Appearance}

All techniques suffer from a certain amount of appearance error due to resampling and rounding in the textures used by the OpenGL renderer to render the novel video sequences. OpenGL requires textures to have dimensions that are a power of 2, this has required some down-sampling of the original images. Another error occurs when texturing a triangle using the original camera images. Although the texture co-ordinates used are corrected for the radial distortion in the image, OpenGL performs a linear interpolation between texture coordinates across a triangle and this will be incorrect for an image with radial distortion. This can be seen in the blurriness of the images produced by the visual hull and the billboard techniques as shown in Figure 2 (d).

In the billboard and view-dependent textured visual hull techniques the images projected onto the surface are those from the nearest two cameras to the virtual camera. These images are transitioned by a simple linear blend. This is virtually guaranteed to generate appearance errors as there is nothing to enforce correspondence between the images, and therefore any disparity between the images will result in a blurring effect across the texture. In high contrast areas this will guarantee that no pixels are similar to the originals and will give very poor appearance.

Where $I_{r}$ has been included in the data set for a reconstruction then the entire image should be free from errors of shape or appearance. This property is called epipole consistency [2]. By looking at the shape and appearance errors present in a view that should be "perfect" in this way, a measure of the avoidable error in the technique can be obtained. From this it can be seen that the implementation of VDVH is furthest away from its best potential performance.

\subsection{Temporal errors}

Matting quality can vary greatly from frame to frame. Figures 8 (e) and (f) show the same player in two consecutive frames demonstrating this effect. As objects change orientation and come into and out of shadow their visual surface properties change which can have an effect on matting quality. Similarly, a fast moving arm may not present sufficient contrast against the background to be picked out by the matting algorithm. In the following frame that same arm could be moving slower and so produce a clear image for the matting algorithm to work on. Such errors will cause limbs to pop in and out of appearance in all of the techniques mentioned.

Billboards suffer greatly from inter-object occlusion. As these occlusions are transient in nature, billboards are particularly susceptible to resultant temporal instabilities. Visual hull and VDVH suffer similarly from inter-object occlusions, although in this case they will tend to cause phantom volumes. These volumes will come into and out of existence over a period of time, and so their results will lead to temporal instability in the reconstruction.

One notable feature of Figure 6 is that reducing the number of cameras increases the stability of the reconstructions. This is due to the fact that all techniques rely on a true surface generating agreement between all cameras. For example if a silhouette is missing from just one camera, then the object will be absent from the reconstruction. If the matting and calibration were perfect then this assumption would be correct. With imperfect matting and calibration this effect can lead to a single bad frame in one camera removing a player from the reconstruction. Obviously as the number of cameras is reduced the chance of disagreement between cameras, and hence the likelihood of this kind of error, is reduced.

\section{Conclusion}

None of the existing techniques provide synthesised video of sufficiently high quality. The technique that gives best performance as the number of available cameras decreases is the VDVH technique. Billboards perform particularly poorly as image misalignment produces errors in shape and appearance. The visual hull technique currently gives the best shape reconstruction due to the error correction mechanism it uses. This mechanism expands the volumes in the reconstruction which leads to misalignment between the images used to texture the surface, causing similar problems to the billboarding technique albeit on a smaller scale. The poor 
scores for the "leave none out" tests indicate that there are many errors in the system which could be corrected. Visual hull is currently the best technique to use for both shape and appearance of reconstruction. VDVH has good potential as a technique due to its lack of degradation with removal of cameras, however the errors in shape currently make it unsuitable for use. Future work will investigate improvements in FVV for sports measured against the baseline performance metrics established in this paper.

\section{Acknowledgements}

This work was supported by the DTI Technology programme project iView: Free-viewpoint video for interactive entertainment production TP/3/DSM/6/I/15515 and EPSRC Grant EP/D033926, lead by BBC Research and Development (http://www.bbc.co.uk/rd/iview). The authors gratefully acknowledge the project partners for providing the sports footage and discussion of the research reported in this paper.

\section{References}

[1] BBC. The Piero System, BBC Production Magic. http://www.bbc.co.uk/rd/projects/virtual/piero.

[2] C Buehler, M Bosse, L McMillan, S Gortler, and $\mathrm{M}$ Cohen. Unstructured lumigraph rendering. In Proceedings ACM SIGGRAPH 28, pages 425-432, 2001.

[3] J. Carranza, C. Theobalt, M. Magnor, and H.-P. Seidel. Free-viewpoint video of human actors. Proceedings ACM SIGGRAPH, 22(3):569-577, 2003.

[4] Y Chuang, B Curless, D Salesin, and R Szeliski. A bayesian approach to digital matting. In Proceedings of IEEE CVPR 2001, volume 2, pages 264-271, December 2001.

[5] K. Connor and I. Reid. A multiple view layered representation for dynamic novel view synthesis. In Proceedings of the 14th British Machine Vision Conference (BMVC), 2003.

[6] P. Debevec, Y. Yu, and G. Borshukov. Efficient viewdependent image-based rendering with projective texturemapping. 9th Eurographics Rendering Workshop, pages 105-116, 1998.

[7] Eye-Vision. Carnegie Mellon goes to the Super Bowl. http://www.ri.cmu.edu/events/sb35/tksuperbowl.html.

[8] J-S. Franco and E. Boyer. Fusion of multi-view silhouette cues using a space occupancy grid. In 10th IEEE International Conference on Computer Vision (ICCV 2005), pages 1747-1753, 2005.
[9] N. Inamoto and H. Saito. Arbitrary viewpoint observation for soccer match video. The 1st European Conference on Visual Media Production (CVMP), pages 21-30, 2004.

[10] T. Kanade, P.W. Rander, and P.J. Narayanan. Virtualized reality: Constructing virtual worlds from real scenes. IEEE Multimedia, 4(1):34-47, 1997.

[11] T. Koyama, I. Kitahara, and Y. Ohta. Live mixedreality $3 \mathrm{~d}$ video in soccer stadium. The 2 nd IEEE and ACM International Symposium on Mixed and Augmented Reality, pages 178-186, 2003.

[12] K Kutulakos. Approximate n-view stereo. In ECCV (1), pages 67-83, 2000.

[13] A. Laurentini. The visual hull concept for silhouette based image understanding. IEEE Transactions on Pattern Analysis and Machine Intelligence, 16(2):150$162,1994$.

[14] W. Lorenson and H. Cline. Marching cubes: A high resolution 3d surface construction algorithm. Computer Graphics, 21(4):163-169, 1987.

[15] W. Matusik, C. Buehler, R. Raskar, S.J. Gortler, and L. McMillan. Image-based visual hulls. Proceedings of ACM SIGGRAPH, pages 369-374, 2000.

[16] G. Miller and A. Hilton. Exact view-dependent visual hulls. In Proceedings of the 18th International Conference on Pattern Recognition (ICPR), 2006.

[17] G Miller, A Hilton, and J Starck. Interactive freeviewpoint video. In Proc. 2nd European Conference on Visual Media Production. IEE, November 2005.

[18] S. Moezzi, L.C. Tai, and P. Gerard. Virtual view generation for $3 \mathrm{~d}$ digital video. IEEE Multimedia, 4(1):18-25, 1997.

[19] H.R. Sheikh and A.C. Bovik. Image information and visual quality. IEEE Transactions on Image Processing, 15(2):430-444, 2006.

[20] J. Starck and A. Hilton. Virtual view synthesis of people from multiple view video sequences. Graphical Models, 67(6):600-620, November 2005.

[21] R. Szeliski. Rapid octree construction from image sequences. CVGIP: Image Understanding, 58(1):23-32, 1993.

[22] S. Vedula, S. Baker, and T. Kanade. Image-based spatiotemporal modeling and view interpolation of dynamic events. ACM Transaction on Graphics, 24(2):240-261, 2005 .

[23] C.L. Zitnick, S. B. Kang, M. Uyttendaele, S. A. J. Winder, and R. Szeliski. High-quality video view interpolation using a layered representation. Proceedings ACM SIGGRAPH, 23:600-608, 2004. 